\title{
Integrated analysis and consistency measurement of bremsstrahlung and charge exchange spectroscopy data for the determination of the ion effective charge ${ }^{a)}$
}

\author{
G. Verdoolaege, ${ }^{1, b)}$ R. Fischer, ${ }^{2}$ and G. Van Oost ${ }^{3}$ \\ ${ }^{1}$ Department of Data Analysis, Ghent University, 9000 Gent, Belgium \\ ${ }^{2}$ Max-Planck-Institut für Plasmaphysik, EURATOM Association, 85748 Garching, Germany \\ ${ }^{3}$ Department of Applied Physics, Ghent University, 9000 Gent, Belgium
}

(Presented 18 May 2010; received 15 May 2010; accepted 7 June 2010; published online 1 October 2010)

\begin{abstract}
In the context of Bayesian probability theory, we discuss a model for estimating the plasma ion effective charge $Z_{\text {eff, integrating data from both bremsstrahlung spectroscopy and individual }}$ impurity concentrations obtained via charge exchange spectroscopy (CXS). The validity of the model, taking into account statistical as well as systematic uncertainties, is shown via the deviance information criterion. The consistency of the continuum and CXS data regarding $Z_{\text {eff }}$ is improved, as measured by the symmetrized Kullback-Leibler divergence and the geodesic distance between the respective $Z_{\mathrm{eff}}$ marginal posterior densities. (C) 2010 American Institute of Physics.

[doi:10.1063/1.3464466]
\end{abstract}

\section{INTRODUCTION}

In this paper, we apply the framework of integrated data analysis (IDA) using Bayesian probability theory ${ }^{1}$ to calculate probabilities associated to the plasma ion effective charge $Z_{\text {eff }}$. There is a long-standing issue of inconsistency due to systematic uncertainties between the $Z_{\text {eff }}$ value assessed from bremsstrahlung measurements (continuum $Z_{\text {eff }}$ ) and the $Z_{\text {eff }}$ calculated from impurity density measurements obtained via charge exchange spectroscopy $(\mathrm{CXS})\left(\mathrm{CX} Z_{\text {eff }}\right)$. This is a general problem observed at various machines. ${ }^{2} \mathrm{~A}$ step toward an initial reconciliation of the two $Z_{\text {eff }}$ estimates through the combined analysis of local continuum and CX data was described in Ref. 1. In the present paper, we build on the work in Ref. 1 by addressing the issues of model selection and consistency measurement. These issues are of fundamental interest in probabilistic data analysis. The purpose of this paper is to demonstrate their relevance to fusion data analysis through the case study of $Z_{\text {eff }}$ estimation.

\section{INTEGRATED $Z_{\text {eff }}$ ESTIMATION}

We discussed the general problem of discrepancy of the continuum and $\mathrm{CX} Z_{\text {eff }}$ in detail in Ref. 1. A simple forward model and an associated probabilistic model were proposed to estimate $Z_{\text {eff }}$ from both the continuum and CX data. Two artificially constructed local plasma quantities $\epsilon$ and $\delta$ were considered as measurements. Apart from $Z_{\text {eff }}$, also the electron density $n_{\mathrm{e}}$ was treated as a quantity of interest due to its strong correlation with $Z_{\text {eff. }}$ The following model was proposed:

\footnotetext{
${ }^{\text {a) }}$ Contributed paper, published as part of the Proceedings of the 18th Topical Conference on High-Temperature Plasma Diagnostics, Wildwood, New Jersey, May 2010.

${ }^{\text {b) }}$ Author to whom correspondence should be addressed. Electronic mail: geert.verdoolaege@ugent.be.
}

$$
\left\{\begin{array}{l}
\epsilon=s_{\epsilon} n_{\mathrm{e}}^{2} Z_{\text {eff }}+\nu_{\epsilon} \\
\delta=s_{\delta} n_{\mathrm{e}}\left(Z_{\text {eff }}-1\right)+\nu_{\delta} \\
n_{\mathrm{e}, \mathrm{L}}=n_{\mathrm{e}}+\nu_{n_{\mathrm{e}}} .
\end{array}\right.
$$

Here, $n_{\mathrm{e}, \mathrm{L}}$ refers to the density measurements, while $\boldsymbol{\nu}$ $\equiv\left[\nu_{\epsilon}, \nu_{\delta}, \nu_{n_{\mathrm{e}}}\right]^{\mathrm{T}}$ is an error term, assumed to be Gaussian with zero correlation. $s_{\epsilon}$ and $s_{\delta}$ are two scale factors that, to a first approximation, summarize the systematic uncertainties. Sufficient information on the scale factors could be obtained by the requirement of consistency between two measurement time slices (levels), each characterized by stationary plasma conditions. The joint and marginal posterior densities were simulated using a Markov chain Monte Carlo (MCMC) algorithm. Good results regarding the estimation of a consistent $Z_{\text {eff }}$ were obtained in Ref. 1 with an accuracy approaching ITER requirements.

In this work, for demonstration purposes we performed the estimation of $Z_{\text {eff }}$ from a synthetic data set (assumed measurement error of $15 \%$ on $\epsilon, 10 \%$ on $\delta$, and $5 \%$ on $n_{\mathrm{e}, \mathrm{L}}$ ). The original and estimated parameter values (for each time slice) are shown in Table I.

\section{MODEL COMPARISON AND CONSISTENCY ANALYSIS}

\section{A. Model comparison}

We next compare the proposed model in Eq. (1) that treats the systematic uncertainties with a simpler model that does not involve the scale factors $s_{\epsilon}$ and $s_{\delta}$ (set equal to 1 ). To evaluate the performance of a model, one needs to weigh the model fit against the model complexity. This has to be done on the basis of the MCMC output and in such a case the deviance information criterion (DIC) is well suited as a model selection tool. The DIC is based on the concept of Bayesian deviance $D(\boldsymbol{\theta})$, defined by ${ }^{3}$ 
TABLE I. A priori chosen and MCMC estimated values for the parameters of interest in the model (1), including $68.3 \%$ credible intervals.

\begin{tabular}{lcccc}
\hline \hline Parameter & $\begin{array}{c}\text { Original } \\
\text { value }\end{array}$ & $\begin{array}{c}\text { Estimated } \\
\text { value }\end{array}$ & $\begin{array}{c}\text { Absolute } \\
\text { error }\end{array}$ & $\begin{array}{c}\text { Relative } \\
\text { error }\end{array}$ \\
\hline$n_{\mathrm{e}, 1}\left(10^{13} \mathrm{~cm}^{-3}\right)$ & 3.24 & 3.25 & \pm 0.05 & $\pm 2 \%$ \\
$n_{\mathrm{e}, 2}\left(10^{13} \mathrm{~cm}^{-3}\right)$ & 4.52 & 4.53 & \pm 0.06 & $\pm 1 \%$ \\
$Z_{\mathrm{eff}, 1}$ & 2.19 & 2.21 & $+0.25 /-0.39$ & $+11 \% /-18 \%$ \\
$Z_{\mathrm{eff}, 2}$ & 2.36 & 2.39 & $+0.20 /-0.38$ & $+8 \% /-16 \%$ \\
$s_{\epsilon}$ & 1.35 & 1.38 & $+0.29 /-0.11$ & $+21 \% /-8 \%$ \\
$s_{\delta}$ & 0.63 & 0.65 & $+0.44 /-0.15$ & $+70 \% /-24 \%$ \\
\hline \hline
\end{tabular}

$$
D(\boldsymbol{\theta})=-2 \ln [p(\boldsymbol{x} \mid \boldsymbol{\theta})]+K,
$$

where $\boldsymbol{x}$ and $\boldsymbol{\theta}$ represent the data and the model parameters, respectively, and $K$ depends only on the data. Clearly, the better the data variability is explained by the model with parameters $\boldsymbol{\theta}$ (i.e., the higher the likelihood) the lower the corresponding deviance. Taking the posterior sample mean $\overline{\boldsymbol{\theta}}$ as an estimator for the parameter vector $\boldsymbol{\theta}$, we have that

$$
\mathrm{DIC} \equiv 2 \overline{D(\boldsymbol{\theta})}-D(\overline{\boldsymbol{\theta}})=\overline{D(\boldsymbol{\theta})}+p_{D}, \quad p_{D} \equiv \overline{D(\boldsymbol{\theta})}-D(\overline{\boldsymbol{\theta}}) .
$$

Here, $p_{D}$ can be seen as a degree of model complexity, measuring the effective number of model parameters. ${ }^{3}$ Models with a smaller DIC should be preferred over others. Using the synthetic data, we obtained a DIC of 225 and a $p_{D}$ of 3.98 for the simpler model that deals only with the statistical sources of uncertainty. For the model including the treatment of systematic uncertainties, the DIC was 30 with a $p_{D}$ of -28 . We can conclude that first, the model with consideration of the scale factors provides a superior explanation for the data. Second, $p_{D}$ for the simpler model is about the true number of parameters because the corresponding posterior is about normal. Third, $p_{D}$ for the model with scale factors is negative, which is due to the considerable skewness of the marginal posteriors for the scale factors.

As a simple example of a comparison with a different model, we discuss the case where, during estimation, the systematic uncertainties are not modeled via multiplicative scale factors, but through an additive term for both the continuum and CX data. Clearly, the latter model is less appropriate to explain our artificial data set that was constructed using the model with scale factors. Indeed, this fact comes out of the analysis in the model comparison phase since the additive model has a DIC of $83\left(p_{D}=5.12\right)$, indicating an inferior model compared to the case with scale factors.

\section{B. Consistency measurement}

In order to get a more clear idea of what has really been gained by modeling the systematic uncertainties, we investigate to what extent the consistency of the continuum and CX data sets has been improved with respect to their predictions of the effective charge. Moreover, such a study can help localize the primary sources of systematic uncertainty. This can be done by observing the change of data consistency when one or more of the nuisance parameters describing systematic uncertainty is left out of the model.

To evaluate the data consistency, we measured the similarity between the marginal posterior densities for $Z_{\text {eff }}$ ob-

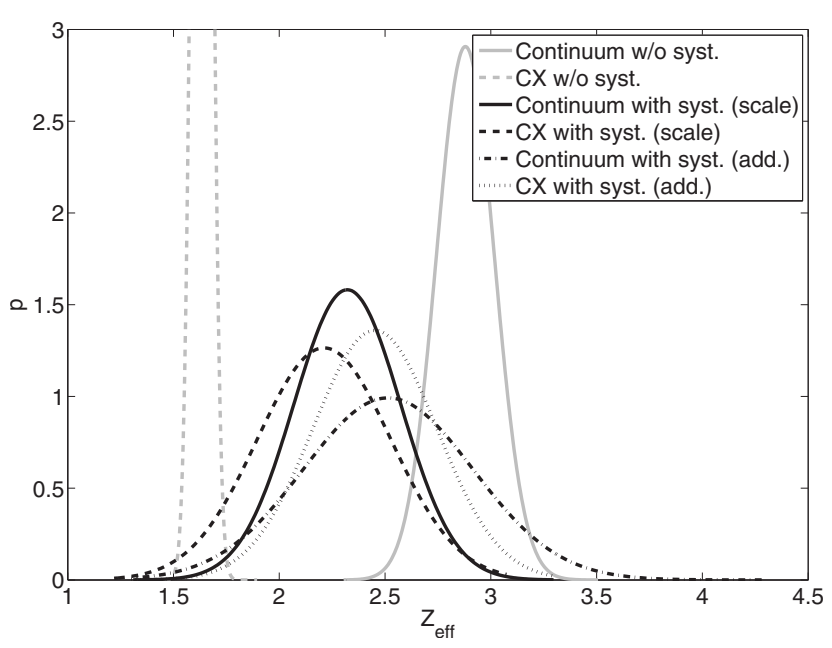

FIG. 1. Normal distributions fit to the marginal posteriors for $Z_{\text {eff }}$ (synthetic data), based on either the continuum or the CX data. The cases are shown without and with treatment of the systematic uncertainties (scale factors or additive terms).

tained from either the continuum or the CX data. We performed this check once using informative prior information on the scale factors and once without taking into account the systematic uncertainties. We first carried out the estimation of the full model (1) at two levels on the artificial data, resulting in comparable estimates of $s_{\epsilon}$ and $s_{\delta}$ as the ones mentioned in Table I. Next, the thus obtained marginal posteriors for the scale parameters were used as prior distributions in the estimation of $Z_{\text {eff }}$ using either the continuum or the CX data in the first time slice. These prior distributions were, in view of their substantial skewness, modeled by generalized extreme value distributions. The resulting continuum and $\mathrm{CX} Z_{\mathrm{eff}}$ marginal posteriors were, computationally conveniently, found to be relatively well described by a normal distribution. Then, the similarity between the continuum and CX marginal was summarized using two probability density similarity measures: the symmetrized Kullback-Leibler divergence (KLD) (Ref. 4) and the Rao geodesic distance (GD) based on the Fisher information as a metric tensor on the manifold of probability density function (PDFs). ${ }^{5}$ Both the KLD and GD can be used as a measure of similarity of the information contained in a PDF, in any application where the resemblance of probabilistic models needs to be assessed. Next, the estimation using either the continuum or CX data in the first time slice was repeated, but keeping the scale factors fixed at 1. Finally, again the KLD and GD between the resulting continuum and CX marginal $Z_{\text {eff }}$ distributions were computed.

Figure 1 presents the thus obtained marginal $Z_{\text {eff }}$ posteriors. In the case of modeled systematic uncertainties (scale factors), the overlap between the continuum and CX distribution is clearly much larger compared to the case without scale factors. In the former case, the relatively large residual statistical uncertainty is due to the uncertainty in the priors on the scale factors. However, note that here data from only one time slice were used, as opposed to two levels for deriving the results in Table I. The difference in overlap is confirmed through the calculation of the respective similarity measures. The symmetrized KLD between the $Z_{\text {eff }}$ posteriors 


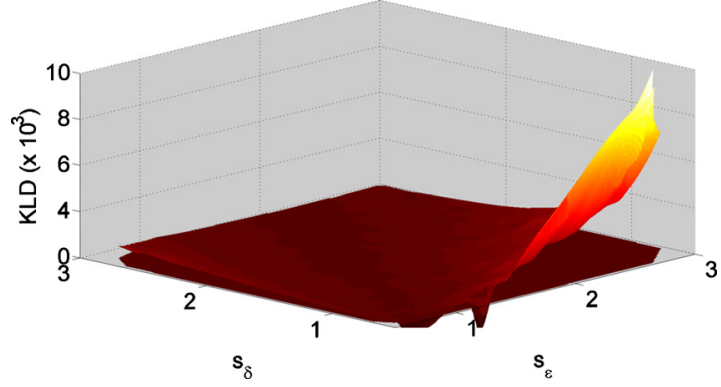

FIG. 2. (Color online) Plot of the KLD between marginal posterior $Z_{\text {eff }}$ distributions based on either continuum or CX data.

obtained from the continuum and CX data in the first time slice, without treatment of the systematic uncertainty, equals 554 while the GD is 7.1. On the contrary, if the systematic uncertainties are modeled and taking into account the informative prior information on the scale factors, we obtain a symmetrized KLD value of 0.26 and a GD of 0.50 . This proves that the consistency of the data, as far as its information content about $Z_{\text {eff }}$ is concerned, improves drastically if we allow for the possibility of a systematic deviation.

Again, if the true model includes scale factors, but instead the model used during estimation contains additive nuisance parameters, we can perform a similar analysis. The resulting marginal $Z_{\text {eff }}$ posteriors are also shown in Fig. 1. The obtained $Z_{\text {eff }}$ probabilities are comparable to the case of the model with scale factors, and the consistency of continuum and CX data is also substantially better (KLD $=0.24, \mathrm{GD}=0.48)$ than in the case where no systematic uncertainties are considered. Still, the model with scale factors is to be preferred over the additive model on the basis of the DIC.

In order to provide a better intuition for the link between the obtained KLD and GD values and the amount of actual data consistency, we performed the following simulation study. Using the values for the density and $Z_{\text {eff }}$ at two levels obtained in Table I, we synthesized a large number of artificial data sets $\left(\epsilon, \delta\right.$, and $\left.n_{\mathrm{e}, \mathrm{L}}\right)$, assuming random values for the scale factors $\left(0.5 \leq s_{\epsilon}, s_{\delta} \leq 3\right)$. For every data set, we carried out the same analysis as described above (systematic uncertainties modeled through scale factors) and we obtained symmetrized KLD and GD values between $Z_{\text {eff }}$ marginal posteriors in the first level. The result for the KLD and GD is displayed in Figs. 2-4, for both the cases with and without treatment of systematic uncertainties. It can be seen that, as expected, the more the scale factors differ from unity, the

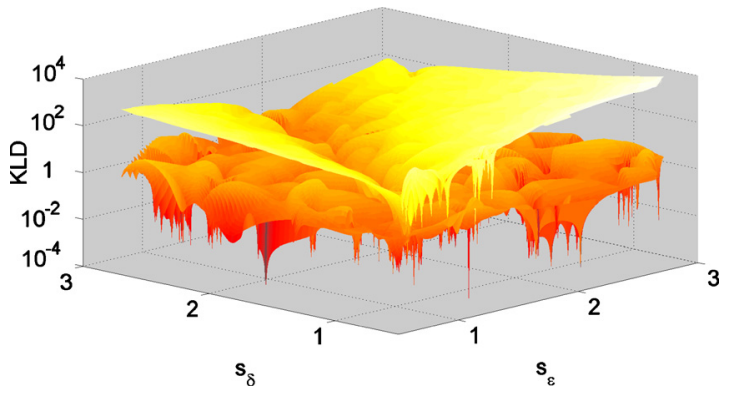

FIG. 3. (Color online) Similar to Fig. 2, but on a logarithmic scale.

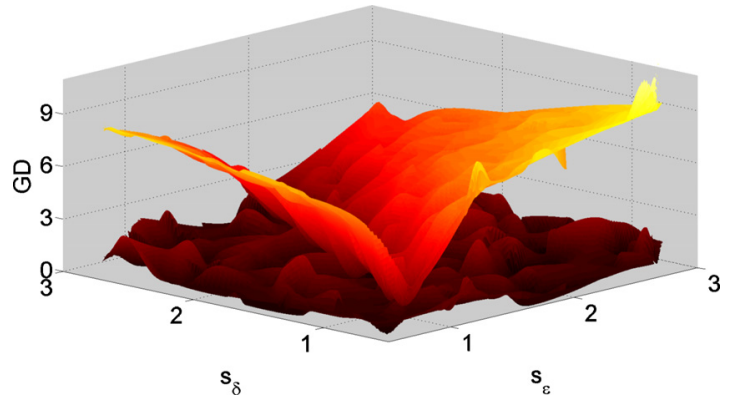

FIG. 4. (Color online) Similar to Fig. 2, but for the GD.

larger the data inconsistency in terms of $Z_{\text {eff }}$ as measured by the KLD and the GD (some values of $s_{\epsilon}$ and $s_{\delta}$ compensate each other). The results from this simulation study can be used as a scale to which newly obtained KLD and GD values can be compared, e.g., using real data. In addition, the KLD seems to be more sensitive to data inconsistency than the GD, but on the other hand in our experiments the GD appeared to be more faithful to an actual measure of data consistency, compared to the KLD. For instance, the huge asymmetry in the KLD values with respect to $s_{\epsilon}$ and $s_{\delta}$ when the scale factors are not included in the model is not well reflected in the size of the systematic uncertainties that were actually introduced in the artificial data. Indeed, as far as $Z_{\text {eff }}$ is concerned, a somewhat less asymmetrical behavior with respect to $s_{\epsilon}$ and $s_{\delta}$ would be expected, as confirmed by the GD measurements in Fig. 4. This finding corresponds to other observations of the KLD and the GD as PDF similarity measures, where it is seen that the GD better matches the actual information content of the distributions. ${ }^{6}$

\section{CONCLUSION}

We have discussed the issues of model selection (using the DIC) and consistency measurement (using the KLD and the GD) in an integrated analysis for the estimation of a consistent $Z_{\text {eff }}$ from both bremsstrahlung and CX impurity density measurements, quantifying systematic uncertainties. In order to evaluate an IDA analysis, it is useful to compare with alternative models (assess model fit and complexity) as well as to analyze the resulting consistency of the data. The methods developed in this work can be applied to issues of inconsistency encountered with other plasma diagnostics as well, for other plasma parameters.

${ }^{1}$ G. Verdoolaege, R. Fischer, G. Van Oost, and JET EFDA Contributors, "Potential of a Bayesian integrated determination of the ion effective charge via bremsstrahlung and charge exchange spectroscopy in tokamak plasmas," submitted to IEEE Trans. Plasma Sci.

${ }^{2}$ M. von Hellermann, Proceedings of the Third Workshop on Fusion Data Processing, Validation and Analysis, Cadarache, 2004.

${ }^{3}$ D. Spiegelhalter, N. Best, B. Carlin, and A. van der Linde, J. R. Stat Soc. Ser. B (Stat. Methodol.) 64, 583 (2002).

${ }^{4}$ S. Kullback, Information Theory and Statistics (Dover, New York, 1968).

${ }^{5}$ S.-I. Amari, O. Barndorff-Nielsen, R. Kass, S. Lauritzen, and C. Rao, Differential Geometry in Statistical Inference, Lecture Notes-Monograph Series Vol. 10 (Institute of Mathematical Statistics, Hayward, CA, 1987), Chap. 5.

${ }^{6}$ G. Verdoolaege and P. Scheunders, "Geodesics on the manifold of multivariate generalized Gaussian distributions with an application to multicomponent texture discrimination," submitted to IEEE Trans. Image Process. 